\title{
Predictors of surgical outcomes of retroperitoneal laparoscopic partial nephrectomy
}

\author{
Carmine Sciorio ${ }^{1}$, Pier Paolo Prontera ${ }^{2}$, Salvatore Scuzzarella ${ }^{1}$, Paolo Verze ${ }^{3}$, Lorenzo Spirito ${ }^{3}$, \\ Lorenzo Romano ${ }^{3}$, Alberto Trinchieri ${ }^{4}$ \\ ${ }^{1}$ Urology Unit, Manzoni Hospital, ASST Lecco, Lecco, Italy; \\ ${ }^{2}$ Urology Unit, S.S. Annunziata Hospital, ASL Taranto, Taranto, Italy; \\ ${ }^{3}$ Department of Urology, University of Naples Federico II, Naples, Italy; \\ ${ }^{4}$ Urology Department, IRCCS Ca' Granda Ospedale Maggiore Policlinico, Milano, Italy.
}

\begin{abstract}
Summary $\quad$ Objectives: To evaluate surgical outcomes partial nephrectomies. in a series of laparoscopic retroperitoneal

Methods: A total of 147 patients who underwent laparoscopic retroperitoneal partial nephrectomy by a single surgeon were evaluated. Pre-operative parameters (body mass index, ASA score, tumour size, cTNM stage, PADUA score risk, surgeon experience) and intraoperative and postoperative outcomes (operative mean time, warm ischemia time, blood loss, transfusion rate, length of hospitalization, and margin-ischaemiacomplications [MIC] success rate) were considered. Results: For 134 patients (91.1\%) the success of the treatment, defined by a MIC = 3, was obtained. When the statistical significance of each of the independent variables was tested, surgeon's experience added statistical significance to the prediction of operative time $(p=0.000)$, warm ischemia time $(p=0.000)$ and blood loss $(p=0.000) ;$ tumour size $(p=0.046)$ to the prediction MIC $(p=0.010)$, operative time $(p=0.000)$, warm ischemia time $(p=0.003)$ and blood loss $(p=0.010)$; ASA score to the length of hospitalization $(p=0.009)$.

Conclusions: Laparoscopic retroperitoneal partial nephrectomy represents an adequate and safe technique for the treatment of $T 1$ renal cancer. Optimal MIC success rate can be achieved, although intraoperative outcomes tend to be related to the learning curve even in a very experienced laparoscopic surgeon. Length of hospitalization depends on general health condition of patients.
\end{abstract}

KEY WORDS: Laparoscopy; Partial nephrectomy; Kidney neoplasms; Retroperitoneal; PADUA score.

Submitted 10 May 2020; Accepted 11 May 2020

\section{INTRODUCTION}

Over the past few years, both robotic and laparoscopic partial nephrectomy (LPN) have emerged as a strong alternative to open partial nephrectomy due to several documented advantages including less blood loss, quicker recovery, less complications (1).

In 2006, Carini et al. presented their successful results of their long-term follow-up study of post-operative outcomes after open partial nephrectomy for pTla tumours (2). The Authors show favourable results as a cancer-specific survival (CSS) of $96.7 \%$ and $94.7 \%$ at, respectively, 5 and 10 years, with a progression-free survival (PFS) of $96 \%$ and $94 \%$ at 5 and 10 years, respectively. Just 1.5\% of patients developed a local recurrence, with no distant metastasis, far from the site of the previous tumorecto- my. Gill et al. demonstrated that LPN was a viable alternative for treating selected renal tumour patients (3). Moreover, in last decades, indications for LPN have progressively extended to tumors of greater diameter and stage (pTlb) (4).

For these reasons, many preoperative score systems have been proposed in order to estimate the pre-operative risk, based on computed tomography (CT) scan and magnetic resonance imaging (MRI). Most commonly used are the PADUA (5) score and the RENAL system (6) score. Both trans and retro peritoneal approach are commonly used by surgeons with the comparable surgical and functional outcomes (7), although the retro peritoneal access, on the one hand guarantees a more restricted working space and a worse exposure of the medial kidney facia, on the other, it allows an immediate access to the renal vessels and reduces the risk of injury to the intra peritoneal organs.

The increasingly extreme use of partial nephrectomy is in terms of the size of the treated masses and of the comorbidity of the patients subjected to this procedure (solitary kidney, synchronous bilateral tumors, etc.) justifies the attention to the oncological and functional result, also in consideration of learning curves. Surgical results were evaluated using MIC-criteria (margin, ischaemia and complications) (8) that anticipated the Trifecta-criteria (9). The only difference between the two score-systems is the length of ischaemia time (20 vs 25 minutes). Herein, we report on postoperative outcomes of a single surgeon, single centre series of retroperitoneal LPN, in order to assess the correlation between preoperative parameters, including the individual nephrometric PADUA score, and post-operative outcomes, including MIC score, in order to provide an evaluation for surgical risk evaluation also related to the surgeon experience.

\section{Patients AND METHOdS}

We designed a monocentric, single surgeon retrospective nonrandomized study. A total of 147 patients, who underwent retroperitoneal LPN for malignancy from March 2014 to April 2018, were enrolled. All the anonymously collected data have been retrospectively evaluated. All procedures were performed by a single surgeon with a 
previous long-lasting experience in renal laparoscopy. Exclusion criteria were as follows: a) distant metastases at the time of the diagnosis; b) previous retroperitoneal surgery. Informed consent was administered and preoperative abdominal CT scan and/or MRI were performed for all patients in order to assess maximum lesion diameter and clinical TNM staging (10). Preoperative PADUA score (5) for each patient was calculated after the CT scan or MRI evaluation performed by two different radiologists, considering the site of the lesion (polar or nonpolar), the extension (exophytic/endophytic), the location at the equator (lateral/medial), the involvement of the renal hilum and the excretory pathway. Tumours were stratified in low (PADUA score: 6-7), medium (PADUA score: 1-2) and high risk (PADUA score: $=/>10$ ). Age, gender, body mass index (BMI), American Society of Anesthesiologists (ASA) score, comorbidities (such as diabetes and hypertension), blood loss, operative time (trocars-in to trocars-out), warm ischemia time, conversion rate to open surgery, pathological stage, tumour histotype according to the classification of the World Health Organization (WHO), nuclear grading according to Fuhrman's classification (11) and surgical margins involvement have been evaluated. Peri and post-operative complications (within the first 30 days) have also been recorded and classified in minor and major according to the Clavien-Dindo system (12) (grade 1-2 and 34 respectively). The surgeon's experience was assessed by dividing surgical procedures into 6 chronological groups according to his learning curve.

Finally, the successful rate for each procedure was estimated using validate M.I.C. system (8) (which considers ischemia time less than 20 minutes, absence of surgical margins involvement and no major post-operative complication). According to this trifecta system, each patient has received from 1 to 3 points.

A descriptive statistical analysis of emerged data was performed. The continuous variables were reported as mean values and standard deviation while the categorical variables were reported as the number of cases $\left(n^{\circ}\right)$ and percentage (\%) and their differences were correlated with the Pearson $\mathrm{x}^{2}$.

Multiple linear logistic regression was used to evaluate the predict effect of a set of pre-operative parameters (age, gender, BMI, ASA, tumour size, TNM stage, PADUA score and surgeon's experience) on different surgical outcomes as MIC, blood loss, transfusion rate, operative time, warm ischemia time and length of hospitalization. All data were analysed using SPSS software and a p-value $<0.05$ was defined as statistically significant.

\section{RESULTS}

A total of 147 patients (101 male and 46 female), who underwent retroperitoneal LPN from 2014 to 2018, were retrospectively enrolled Mean age was 63 years (range 37-74).

Table 1 shows the baseline cohort characteristics and preoperative data. The assessed mean diameter of renal lesions at pre-operative CT scan was $3.55 \mathrm{~cm}+/-1.65$. Application of PADUA score system allow to stratify the surgical risk as follows: 79 (54\% of the population) as low risk, 51 as
Table 1.

Baseline cohort characteristics and preoperative evaluations (147 pts).

\begin{tabular}{|c|c|}
\hline Median age, years & $63(37.64)$ \\
\hline$\overline{\mathrm{M} / \mathrm{F} \text { ratio (pts) }}$ & $101 / 46$ \\
\hline BMI $\left(\mathrm{kg} / \mathrm{m}^{2)}\right.$ & $25.9 \pm 3.2$ \\
\hline Right/left side (pts) & $83(57 \%) / 64(43 \%)$ \\
\hline Average volume of lesions (radiological evaluation) & $3.55 \pm 1.65 \mathrm{~cm}$ \\
\hline \multicolumn{2}{|l|}{ PADUA score } \\
\hline - Low & 6-7: 79 pts (54\%) \\
\hline - Intermediate & 8-9: 51 pts (35\%) \\
\hline - High & $\geq 10: 17$ pts (11\%) \\
\hline Tumor site (polar/not polar) & 99 pts. (67\%)/48 pts. (33\%) \\
\hline \multicolumn{2}{|l|}{ Tumor site (exophytic/not exophytic/completely } \\
\hline Tumor site (lateral/medial) & 90 pts (61\%)/57 pts (39\%) \\
\hline Renal sinus involvement (yes/no) & 8 pts (5\%)/139 pts (95\%) \\
\hline Excretory system involvement (yes/no) & 6 pts (4\%)/141 pts (96\%) \\
\hline
\end{tabular}

\section{Table 2.}

Intraoperative parameters of the cohort (147 pts).

\begin{tabular}{|lc|}
\hline Average operative time (min) & $118 \pm 35$ \\
\hline Warm ischemia (pts) & $82(56 \%)$ \\
- no ischemia & $51(35 \%)$ \\
$-<20$ minutes & $10(7 \%)$ \\
$-20-29$ minutes & $3(2 \%)$ \\
$->30$ minutes & $6.84 \pm 8.74$ \\
\hline Average time of warm ischemia (min) & $236 \pm 186$ \\
\hline Average blood loss (ml) & $6.01 \pm 5.43$ \\
\hline Average hospital stay (day) & $0.16 \pm 0.65$ \\
\hline Average $n^{\circ}$ of transfusions per patient & $7.5 \%(11 / 147)$ \\
\hline Percentage of transfused patients & $1.4 \%(2 / 147)$ \\
\hline Percentage of major complications & \\
\hline
\end{tabular}

intermediate risk (35\%) and 17 as high risk (11\%). Intraoperative and postoperative results are shown in Table 2. In 82 cases (56\%) no ischemia was performed, whereas 64 patients received some warm ischemia time. In detail, 51 patients (35\%) underwent less than 20 minutes warm ischemia, 10 (7\%) from 20 to 29 minutes and $3(2 \%)$ more than 30 minutes of warm ischemia.

The global mean time of warm ischemia was $6.84+$ /8.74 minutes. Peri-operative complication rate was $1.4 \%$ and there was no case of conversion to open surgery. Average hospitalization time was 6.01 +/- 5.43 days.

Pathological analysis of the lesions shows a prevalence of pTla stage tumours (64\%), followed by pT1b (19.7\%). Pathological stages pT2 and pT3 all together represent $2.7 \%$ of the cohort and 20 lesions (13.6\%) appear to be no-clear cells tumours (CCT). No positive surgical margin was observed.

In our purpose, the success of the treatment was defined by $\mathrm{MIC}=3$, and it was obtained in 134 patients (91.1\%). At multiple linear logistic regression PADUA score, TNM stage (Table 4), tumour size by pre-operative CT, BMI, ASA and surgeon's experience predicted the effect of MIC success ( $p=0.034)$, operative time $(p=0.000)$, warm ischemia time $(p=0.000)$, blood loss $(p=0.000)$ and length of hospitalization $(p=0.002)$. Coefficient of determination R2 explain 10.7\%, 34.7\%, 26.7\%, 23.4\% and $16.2 \%$ of the variability of MIC success, operative time, 
Table 3.

Patient characteristics with and without MIC success (defined as $M I C=3$ ).

\begin{tabular}{|lccc|}
\hline Patients characteristics & MIC success (134 pts) & MIC failure (13 pts) & P-value \\
\hline Average volume of lesions & & & \\
(radiological evaluation) & $3.38 \pm 1.58$ & $5.30 \pm 1.34$ & 0.000 \\
\hline PADUA score & Pts: & Pts: & \\
$-6-7$ & 75 & 4 & 0.006 \\
$-8-9$ & 47 & 4 & \\
$-\geq 10$ & 12 & 5 & \\
\hline Tumor site & Pts: & Pts: & \\
- Exophytic & 80 & 2 & 0.007 \\
- Not exophytic & 47 & 9 & \\
- Completely endophytic & 7 & 2 & \\
\hline pTNM & Pts: & Pts: & \\
- pT1a & 90 & 4 & 0.000 \\
- pT1b & 20 & 9 & \\
- pT2 & 2 & 0 & \\
- pT3 & 2 & 0 & \\
- Others & 20 & 0 & \\
\hline Tumor size (histopathological evaluation) & $3.08 \pm 1.5 \mathrm{~cm}$ & $4.97 \pm 1.47 \mathrm{~cm}$ & 0.000 \\
\hline Average time of warm ischemia (min) & $5.16 \pm 6.96$ & $24.07 \pm 6.14$ & 0.000 \\
\hline Average operative time (min) & $115 \pm 32$ & $151 \pm 51$ & 0.000 \\
\hline Blood loss (ml) & $222 \pm 155$ & $385 \pm 360$ & 0.002 \\
\hline
\end{tabular}

Table 4.

Correlation between surgical outcomes and pathological stage.

\begin{tabular}{|lcccccc|}
\hline & pT1a & pT1b & pT2 & pT3 & 0thers & P-value \\
\hline Mean PADUA score & $7.41 \pm 1.42$ & $8.48 \pm 1.7$ & $9.50 \pm 3.53$ & $8 \pm 0.0$ & $7.35 \pm 1.38$ & 0.000 \\
\hline $\begin{array}{l}\text { Average time } \\
\text { of ischemia }\end{array}$ & $6.01 \pm 7.71$ & $11.62 \pm 11.45$ & 0 & $9.5 \pm 13.43$ & $4.25 \pm 6.4$ & 0.011 \\
\hline $\begin{array}{l}\text { Pathological } \\
\text { tumor size (cm) }\end{array}$ & $2.78 \pm 1.07$ & $4.71 \pm 1.66$ & $5.25 \pm 5.3$ & $5.75 \pm 4.59$ & $2.88 \pm 1.24$ & 0.000 \\
\hline MIC=3 (pts) & $90 / 94$ & $20 / 29$ & $2 / 2$ & $2 / 2$ & $20 / 20$ & 0.000 \\
\hline
\end{tabular}

warm ischemia time, blood loss and length of hospitalization. When the statistical significance of each of the independent variables was tested, surgeon's experience added statistical significance to the prediction of operative time $(\mathrm{p}=0.000)$, warm ischemia time $(\mathrm{p}=0.000)$ and blood loss ( $\mathrm{p}=0.000)$; tumour size ( $\mathrm{p}=0.046)$ to the prediction MIC $(p=0.010)$, operative time $(p=0.000)$, warm ischemia time ( $\mathrm{p}=0.003)$ and blood loss $(\mathrm{p}=0.010)$; ASA score to the length of hospitalization ( $\mathrm{p}=0.009$ ).

For 13 patients $(8.9 \%)$ with $\mathrm{MIC}<3$, failure was associated in a statistically significant way to lesion diameter $(\mathrm{p}=0.000)$, TNM classification $>/=\mathrm{pTlb}(72.7 \%$ vs 95.7\%) $(\mathrm{p}=0.000)$, high risk calculated by PADUA score system $(\mathrm{p}=0.006)$ (Table 3$)$. Moreover, for all patients with MIC $<3$ we registered significantly longer times of warm ischemia $(p=0.000)$, longer operating times $(p=$ $0.000)$ and greater blood loss $(p=0.002)$. For all no-CCT MIC was 3, with a successful rate of $100 \%$.

\section{Discussion}

The main goal of the present study was to evaluate the MIC score rate in our series of patients treated by retroperitoneal LPN.

Secondary endpoints were the assessment of the correlation between pre-operative parameters, as PADUA score, and perioperative and postoperative outcomes. The successful rate obtained was elevate (MIC $=3$ was obtained in $91,1 \%$ of patients), comparable to that of Gill's group (13) and to that of other robotic case studies (14). Evaluation of some recent series excluded patients who had not undergone ischemia. By applying this criterion, our success rate would drop, although it should be underlined that in our series ischemia was not used even in many cases with high PADUA score and large diameter tumors. The exclusion of these patients from the study would be penalizing for the evaluation of our results. On the other hand, the MIC evaluation resulted in a "flattening" of the results range upwards. For this reason, in our opinion, the indicators of surgical success should be more stratified, for example by rewarding the absence of ischemia or very low ischemia times ( $<10$ minutes).

It is interesting to note that the learning curve for the examined procedure is long even for an experienced surgeon. In fact, the results obtained by a single surgeon, already an expert at the beginning of this experience, show a specific trend, with a significant improvement in perioperative and postoperative outcomes during the study period. However, the results in terms of MIC are not significantly related to the experience of the surgeon, who, when he has sufficient initial experience in renal laparoscopy, is able to ensure the surgical success of the procedure even with longer operating times and a higher blood loss and transfusion rate.

On the other hand, in the initial phase of the experience the surgeon tends to select cases with lower PADUA score (6-7), while in the continuation of the study were then considered more complex cases with higher PADUA score (>10). More studies are needed to better investigate these relationships and provide the surgeon, both expert and on learning curve, with effective predictive tools to obtain the best results in terms of operative and oncological outcomes.

The use of the traditional laparoscopic technique for partial retroperitoneal nephrectomy has disadvantages and advantages over the robot-assisted technique. Numerous studies have compared the results of traditional laparoscopic partial nephrectomy (LPN) with those of roboticassisted partial nephrectomy (RAPN). Transperitoneal and retroperitoneal RAPN are equally effective and safe in terms of warm ischemia time, estimated blood loss, rate of conversion and complications and positive surgical margins (15). In particular, retroperitoneal RALPN proved to be an excellent option for posterior and lateral tumors with reduced operational times and shortened lenghts of stay (16).

Some Authors reported that RAPN has short operating and ischemia times and less blood loss compared with LPN $(17,18)$, although other studies have shown no differences in terms of operative time, warm ischemia time, estimated blood loss and lenght of hospital stay $(19,20)$. A meta-analysis showed equivalent peri-operative outcomes of LPN and RAPN, which added the advantage of a shorter warm ischaemia time (21).

On the other hand, LPN implies lower healthcare costs and use of sutureless technique can reduce warm ischemia and operative time $(22,23)$.

At the moment, the two techniques represent excellent 
alternatives to the in the management of moderate to complex renal tumours with high PADUA scores.

\section{Conclusions}

Retroperitoneal LPN represents an adequate and safe technique for the treatment of $\mathrm{Tl}$ renal cancer. Optimal MIC success rate can be achieved, although intraoperative outcomes tend to progressively improve during the learning curve even in a very experienced surgeon. Length of hospitalization depends on general health condition.

\section{References}

1. de Castro Abreu AL, Cacciamani G, Gill IS. Partial Nephrectomy. In: Sotelo R., Arriaga J, Aron M. (eds) Complications in Robotic Urologic Surgery. Springer, 2018; pp. 163-173.

2. Carini M, Minervini A, Masieri L, et al. Simple enucleation for the treatment of PT1 a renal cell carcinoma: our 20-year experience. Eur Urol. 2006; 50:1263-8.

3. Gill IS, Desai MM, KaoukJH, et al. Laparoscopic partial nephrectomy for renal tumor: duplicating open surgical techniques. J Urol. 2002; 167:469-7.

4. Ljungberg B, Albiges L, Bensalah K, et al. EAU Guidelines for Renal Cell Carcinoma. Edn. presented at the EAU Annual Congress Copenhagen 2019. ISBN 978-94-92671-04-2.

5. Ficarra V, Novara G, Secco S, et al. Preoperative aspects and dimensions used for an anatomical (PADUA) classification of renal tumors in patients who are candidates for nephron-sparing surgery. Eur Urol, 2009; 56:786-793.

6. Kutikov A, Uzzo RG. The R.E.N.A.L. nephrometry score: a comprehensive standardized system for quantitating renal tumor size, location and depth. J Urol. 2009; 182:844-53.

7. Marszalek M, Chromecki T, Al-Ali BM, et al. Laparoscopic partial nephrectomy: a matched-pair comparison of the transperitoneal versus the retroperitoneal approach. Urology. 2011; 77:109-13.

8. TNM classification of malignant tumors. UICC International Union Against Cancer. $7^{\text {th }}$ ed. Brierley J.D., Gospodariwicz M., Wittekind C. (eds). Wiley-Blackwell, 2009.

9. Buffi N, Lista G, Larcher A, et al. Margin, ischemia and complications (MIC) score in partial nephrectomy: a new system for evaluating achievement of optimal outcomes in nephron-sparing surgery. End Urol. 2012; 62:617-8.

10. Hung AJ, Cai J, Simmons MN et al. "Trifecta" in partial nephrectomy. J Urol. 2013; 189:36-42.

11. Fuhrman SA, Lasky LC, Limas C. Prognostic significance of morphologic parameters in renal cell carcinoma. Am J Surg Pathol. 1982; 6:655-63.

12. Dindo D, Demartines N, Clavien PA. Classification of surgical complications: a new proposal with evaluation in a cohort of 6336 patients and results of a survey. Ann Surg. 2004; 240:205-13.

13. Hung AJ, Cai J, Simmons MN, Gill IS. "Trifecta" in partial nephrectomy. J Urol. 2013; 189:36-42.

14. Bianchi L, Schiavina R, Borghesi M, et al. Which patients with clinical localized renal mass would achieve the trifecta after partial nephrectomy? The impact of surgical technique. Minerva Urol Nefrol. 2019 Oct 10. doi: 10.23736/S0393-2249.19.03485-4. [Epub ahead of print]
15. Xia L, Zhang X, Wang X, et al. Transperitoneal versus retroperitoneal robot-assisted partial nephrectomy: A systematic review and meta-analysis. Int J Surg. 2016; 30:109-15.

16. Laviana AA, Tan HJ, Hu JC, et al. Retroperitoneal versus transperitoneal robotic-assisted laparoscopic partial nephrectomy: a matched-pair, bicenter analysis with cost comparison using timedriven activity-based costing. Curr Opin Urol. 2018; 28:108-114.

17. Pierorazio PM, Patel HD, Feng T, et al. Robotic-assisted versus traditional laparoscopic partial nephrectomy: comparison of outcomes and evaluation of learning curve. Urology. 2011; 78:813-9.

18. Wang Y, Ma X, Huang Q, et al. Comparison of robot-assisted and laparoscopic partial nephrectomy for complex renal tumours with a RENAL nephrometry score $\geq 7$ : peri-operative and oncological outcomes. BJU Int. 2016; 117:126-30.

19. Masson-Lecomte A, Bensalah K, Seringe E, et al. A prospective comparison of surgical and pathological outcomes obtained after robot-assisted or pure laparoscopic partial nephrectomy in moderate to complex renal tumours: results from a French multicentre collaborative study. BJU Int. 2013; 111:256-63.

20. Simsek A, Yavuzsan AH, Colakoglu Y, et al. Comparison of robotic and laparoscopic partial nephrectomy for small renal tumours. Arch Ital Urol Androl. 2017; 89:93-96.

21. Zhang X, Shen Z, Zhong S, et al. Comparison of peri-operative outcomes of robot-assisted vs laparoscopic partial nephrectomy: a meta-analysis. BJU Int. 2013; 112:1133-42.

22. Corongiu E, Grande P, Di Santo A, et al. Safety and efficacy of retroperitoneal sutureless zero ischemia laparoscopic partial nephrectomy for low nephrometry score masses. Arch Ital Urol Androl. 2019; 91:157-162.

23. Tiscione D, Cai T, Luciani LG, et al. Sutureless laparoscopic partial nephrectomy using fibrin gel reduces ischemia time while preserving renal function. Arch Ital Urol Androl. 2019; 91:30-34.

\section{Correspondence \\ Carmine Sciorio, MD (Corresponding Author) \\ carmine.sciorio@gmail.com \\ Salvatore Scuzzarella, MD \\ s.scuzzarella@libero.it \\ ASST “A. Manzoni” Hospital - Lecco (Italy}

Pier Paolo Prontera, MD

pierpaolo.prontera@virgilio.it

ASL-TA "S:S. Annunziata Hospital - Taranto (Italy)

Paolo Verze, MD

pverze@gmail.com

Lorenzo Spirito, MD

lorenzospirito@msn.com

Lorenzo Romano, MD

loryromano@hotmail.it

Università Federico II - Napoli (Italy)

Alberto Trinchieri, MD

alberto.trinchieri@gmail.com

Urology Department, IRCCS Ca' Granda Ospedale Maggiore Policlinico, Milano (Italy) 